\title{
Improved Treatment of Exact Exchange in Quantum ESPRESSO is
}

\author{
Taylor A. Barnes ${ }^{\mathrm{a}, *}$, T. Kurth ${ }^{\mathrm{a}}$, P. Carrier $^{\mathrm{b}}$, N. Wichmann ${ }^{\mathrm{b}}$, David \\ Prendergast $^{\mathrm{c}}$, Paul R. C. Kent ${ }^{\mathrm{d}, \mathrm{e}}$, Jack Deslippe ${ }^{\mathrm{a}}$ \\ ${ }^{a}$ NERSC, Lawrence Berkeley National Laboratory, Berkeley, CA 94720, USA \\ ${ }^{b}$ Cray Inc., Saint Paul, MN 55101, USA \\ ${ }^{c}$ The Molecular Foundry, Lawrence Berkeley National Laboratory, Berkeley, CA 94720, \\ USA \\ ${ }^{d}$ Center for Nanophase Materials Sciences, Oak Ridge National Laboratory, Oak Ridge, \\ TN 37831, USA \\ ${ }^{e}$ Computer Science and Mathematics Division, Oak Ridge National Laboratory, Oak \\ Ridge, TN 37831, USA
}

\begin{abstract}
We present an algorithm and implementation for the parallel computation of exact exchange in Quantum ESPRESSO (QE) that exhibits greatly improved strong scaling. QE is an open-source software package for electronic structure calculations using plane wave density functional theory, and supports the use of local, semi-local, and hybrid DFT functionals. Wider application of hybrid functionals is desirable for the improved simulation of electronic band energy alignments and thermodynamic properties, but the computational complexity of evaluating the exact exchange potential limits the practical application of hybrid functionals to large systems and requires efficient implementations. We demonstrate that existing implementations of hybrid DFT that utilize

\footnotetext{
« This manuscript has been authored by UT-Battelle, LLC under Contract No. DEAC05-00OR22725 with the U.S. Department of Energy. The United States Government retains and the publisher, by accepting the article for publication, acknowledges that the United States Government retains a non-exclusive, paid-up, irrevocable, worldwide license to publish or reproduce the published form of this manuscript, or allow others to do so, for United States Government purposes. The Department of Energy will provide public access to these results of federally sponsored research in accordance with the DOE Public Access Plan (http://energy.gov/downloads/doe-public-access-plan).

${ }^{*}$ Corresponding author. E-mail address: tbarnes@lbl.gov
}

Preprint submitted to Computer Physics Communications

January 7, 2017

(C) 2017. This manuscript version is made available under the Elsevier user license http://www.elsevier.com/open-access/userlicense/1.0/ 
a single data structure for both the local and exact exchange regions of the code are significantly limited in the degree of parallelization achievable. We present a band-pair parallelization approach, in which the calculation of exact exchange is parallelized and evaluated independently from the parallelization of the remainder of the calculation, with the wavefunction data being efficiently transformed on-the-fly into a form that is optimal for each part of the calculation. For a 64 water molecule supercell, our new algorithm reduces the overall time to solution by nearly an order of magnitude.

Keywords: hybrid DFT; QuantumESPRESSO; scalability

\section{Introduction}

The central computational problem in the fields of chemistry and materials science is the accurate and efficient representation and evaluation of the quantum mechanical interactions that govern the properties of complex systems. One of the most important tools for addressing this problem is density functional theory (DFT), in which the interactions between electrons are expressed by a functional of the electronic density [1, 2]. DFT has been successfully applied in a vast array of studies, including characterization of the adsorption properties of porous materials [3], prediction of the thermodynamic and optical properties of new materials [4], simulation of X-ray absorption spectra [5], and investigation of the binding energies of biomolecules [6].

Despite these successes, DFT suffers certain known limitations that result from the difficulty of constructing accurate functionals. In particular, the local and semi-local functionals most commonly used for the study of condensed-phase systems include approximations to static exchange interactions that tend to cause excessive delocalization of the electrons. This in turn results in underestimation of reaction barriers, condensed phase band gaps, and charge transfer excitation energies $[7,8]$.

To a certain extent, these issues can be addressed through the use of hybrid functionals, such as HSE [9, 10], B3LYP [11], and PBE0 [12], which incorporate some amount of exact exchange. Relative to local and semi-local functionals, hybrid functionals have been demonstrated to produce a more accurate degree of charge localization $[13,14]$, improve the accuracy of band gaps $[13,15,16]$ and polarizabilities [17], yield structural properties that 
are more consistent with experiment [15, 18, 19], and enable more accurate simulation of the degree of dissociation of ionic species [20].

A major challenge to the use of hybrid functionals is the large computational cost associated with the calculation of the exact exchange potential, particularly in the case of plane wave implementations which cannot take advantage of the neglect of exchange matrix elements whose basis functions do not overlap, as employed in localized orbital representations. Although considerable progress has been made in recent years [21, 22, 23, 24], hybrid functionals remain prohibitively expensive for many problems. In order to achieve reasonable time-to-solution, hybrid DFT calculations on non-trivial systems typically necessitate the use of large numbers of processors; however, achieving good strong scaling in this regime is algorithmically challenging.

This paper presents a "band-pair" approach to the parallelization of the calculation of exact exchange within the QuantumESPRESSO (QE) software suite [25]. A brief overview of the theory underlying hybrid functionals is provided in Sec. 1.1. In Sec. 2.1 we outline the existing implementation of hybrid DFT in QE, and in Sec. 2.2 identify the primary issues limiting the performance. The band-pair parallelization algorithm is described in Sec. 3.1. In Sec. 3.2, we demonstrate the effectiveness of a parallelization strategy in which the calculation of the exact exchange potential is parallelized independently of the parallelization of the other regions of the code, which is made possible through an efficient algorithm for on-the-fly transformation of the main QE data structures. The details of this data structure transformation are provided in Sec. 3.3. Finally, the results in Sec. 4 demonstrate that our modifications greatly improve the strong scaling performance of the code, enabling the application of hybrid functionals to significantly larger systems than was previously possible within reasonable timescales.

\subsection{KS Theory}

The purpose of a self-consistent field (SCF) calculation in QE is to identify the ground or excited-state wavefuction that satisfies the Kohn-Sham equations,

$$
\hat{H}_{\mathrm{KS}}[\rho ; \mathbf{r}] \psi_{i}(\mathbf{r})=\epsilon_{i} \psi_{i}(\mathbf{r}),
$$

where $\psi_{i}$ is the wavefunction of the $i$ th electronic band, $\hat{H}_{\mathrm{KS}}[\rho ; \mathbf{r}]$ is the KS Hamiltonian, $\epsilon_{i}$ is the eigenvalue of the $i$ th band, and $\rho$ is the electronic 
density,

$$
\rho(\mathbf{r})=\sum_{i=1}^{n_{\text {occ }}}\left|\psi_{i}(\mathbf{r})\right|^{2},
$$

where $n_{\text {occ }}$ is the number of occupied bands. Because the Kohn-Sham Hamiltonian is implicitly dependent upon the wavefunction, practical solution of Eq. 1 is obtained through self-consistent convergence of nested loops that iterate over the Fock operator and the charge density, respectively. Each iteration of the charge density is updated through an iterative Davidson diagonalization algorithm.

In atomic units, the KS Hamiltonian takes the form

$$
\hat{H}_{\mathrm{KS}}[\rho ; \mathbf{r}]=-\frac{1}{2} \nabla^{2}+v_{\mathrm{KS}}[\rho ; \mathbf{r}]+\alpha \hat{K}\left[\left\{\psi_{j}\right\} ; \mathbf{r}\right],
$$

where $\alpha$ is the fraction of exact exchange used. In the case of local or semilocal DFT functionals, $\alpha=0$ and the Hamiltonian becomes functionally dependent only on the electronic density, The local or semi-local contribution to the Hamiltonian is

$$
v_{\mathrm{KS}}[\rho ; \mathbf{r}]=v_{\mathrm{ext}}(\mathbf{r})+v_{\mathrm{J}}[\rho ; \mathbf{r}]+(1-\alpha) v_{\mathrm{xc}}[\rho ; \mathbf{r}],
$$

where $v_{\text {ext }}(\mathbf{r})$ is the external potential, which typically corresponds to the Coulomb potential of the nuclei, $v_{\mathrm{xc}}[\rho ; \mathbf{r}]$ is the local or semi-local approximation to the exchange-correlation potential, and $v_{J}[\rho ; \mathbf{r}]$ is the Coulomb potential resulting from the mean-field electron density,

$$
v_{\mathrm{J}}[\rho ; \mathbf{r}]=\int \frac{\rho\left(\mathbf{r}^{\prime}\right)}{\left|\mathbf{r}^{\prime}-\mathbf{r}\right|} d \mathbf{r}^{\prime} .
$$

The expression $\hat{K}\left[\left\{\psi_{j}\right\} ; \mathbf{r}\right]$ in the last term of 3 is the exact exchange operator. Its action on the wavefunction is

$$
\left(\hat{K}\left[\left\{\psi_{j}\right\} ; \mathbf{r}\right] \psi_{i}\right)(\mathbf{r})=-\sum_{j=1}^{n_{o c c}} k_{i j}(\mathbf{r}) \psi_{j}(\mathbf{r})
$$

where

$$
k_{i j}(\mathbf{r})=\int \frac{\psi_{j}^{*}\left(\mathbf{r}^{\prime}\right) \psi_{i}\left(\mathbf{r}^{\prime}\right)}{\left|\mathbf{r}^{\prime}-\mathbf{r}\right|} d \mathbf{r}^{\prime} .
$$


In Eq. 7 and throughout this paper we use the generalized k-point case. Extension of our algorithm to the gamma-point only case, in which the wavefunctions are represented as real, is trivial and has been implemented. In plane wave implementations, the convolution which defines $k_{i j}(\mathbf{r})$ is typically calculated through the use of a product of Fourier transforms, exploiting the Fast Fourier Transform (FFT) algorithm:

$$
k_{i j}(\mathbf{r})=\mathrm{FFT}^{-1}\left[c(\mathbf{g}) \mathrm{FFT}\left[\psi_{j}^{*}\left(\mathbf{r}^{\prime}\right) \psi_{i}\left(\mathbf{r}^{\prime}\right)\right]\right],
$$

where

$$
c(\mathbf{g})=\mathrm{FFT}\left[\frac{1}{\left|\mathbf{r}^{\prime}-\mathbf{r}\right|}\right] .
$$

The primary cost of a hybrid DFT calculation is the application of the exact exchange operator $\hat{K}\left[\left\{\psi_{j}\right\} ; \mathbf{r}\right]$. Application of $\hat{K}\left[\left\{\psi_{j}\right\} ; \mathbf{r}\right]$ to all of the occupied wavefunctions requires the computation of $n_{\text {occ }}^{2}$ integrals. Each integral is computed through the use of FFTs that are performed numerically over a grid that spans the entire simulation cell; thus, the cost of each individual FFT scales with $\mathcal{O}\left(n_{\mathrm{pw}} \log \left[n_{\mathrm{pw}}\right]\right)$, where $n_{p w}$ is the number of plane waves. As a result, the cost of calculating the exact exchange component of $\hat{H}_{\mathrm{KS}} \psi_{i}\left[\left\{\psi_{j}\right\} ; \mathbf{r}\right]$ tends to exhibit at best $\mathcal{O}\left(n_{\mathrm{occ}}^{2} n_{\mathrm{pw}} \log \left[n_{\mathrm{pw}}\right]\right)$ scaling [21]. By contrast, the cost of evaluating the other contributions to $\hat{H}_{\mathrm{KS}} \psi_{i}\left[\left\{\psi_{j}\right\} ; \mathbf{r}\right]$ tend to scale as $\mathcal{O}\left(n_{\mathrm{occ}} n_{\mathrm{pw}} \log \left[n_{\mathrm{pw}}\right]\right)$ or better.

\section{Methods}

\subsection{Overview of Existing Parallelization}

In Sec. 1.1, it was shown that plane wave DFT simulations typically require the computation of many FFTs. As a result, the most basic level of parallelization employed by QE is plane wave parallelization. In this approach, the plane waves that are used to represent $\psi$ are distributed across processes, with all of the processes simultaneously contributing to the calculation of each FFT. QE includes an algorithm for parallel plane wave calculations that can use external libraries for serial FFTs or, alternatively, an internal version of the FFTW[28] library.

Another approach to parallelization in QE is band parallelization, which involves assigning the work of multiple FFTs to be simultaneously performed by different groups of processes. QE 5.2.0 supports two mutually exclusive methods for band parallelization: (1) "task group" parallelization and (2) 
"band group" parallelization. Although similar in purpose, these two methods affect different regions of the code, and cannot be switched between during runtime. The QE SCF code can be regarded as consisting of two different regions: an exact exchange region that is responsible for the calculation of the exact exchange contributions to the Hamiltonian, energy, and other physical properties of the system, and a local region that is responsible for all other tasks. Task group parallelization only enables parallelization over FFTs in the local regions of the code, while band group parallelization only enables parallelization over FFTs in the exact exchange regions of the code.

Band group parallelization is designed to enhance the performance of vexx_k and vexx_gamma, the subroutines that calculate $\left(\hat{K}\left[\left\{\psi_{j}\right\} ; \mathbf{r}\right] \psi_{i}\right)(\mathbf{r})$ in the k-point and gamma-point only cases, respectively. Algorithm 1 outlines a simplified version of the QE 5.2.0 implementation of vexx_k. The algorithm consists of a double loop over bands $i$ and $j$, with the inner loop being parallelized with respect to band groups. When band groups are used, each process is assigned to a band group, and each band group is assigned a set of bands in the range jbnd_start:jbnd_end. Across all band groups, the values of $j b n d \_s t a r t: j b n d \_e n d$ span the range $1: n_{\text {bnd }}$, where $n_{\text {bnd }}$ is the total number of eigenvectors computed by the iterative Davidson diagonalization algorithm. The outer loop of Algorithm 1 is performed over the total number of bands that have not converged for a given step of the diagonalization algorithm, $n_{\text {uncov }}$. Only the work in the inner loop of Algorithm 1 is parallelized between band groups, with all other work being duplicated across band groups. Within each band group, individual FFTs are parallelized over plane waves; thus, the number of band groups determines the degree to which the calculation uses parallelization over plane waves vs. parallelization over bands. A calculation using a single band group is parallelized exclusively over plane waves, while a calculation using a number of band groups equal to the number of processes is parallelized exclusively over bands. The number of band groups used by a calculation is set at runtime using the -nbgrp option, which defaults to 1 .

\subsection{Performance Issues}

As described in Sec. 2.1, QE offers multiple approaches for parallelizing the calculation of exact exchange, with the number of task groups or band groups controlling the degree to which the parallelization is performed over plane waves or over bands. Fig. 1 illustrates that when using large numbers 


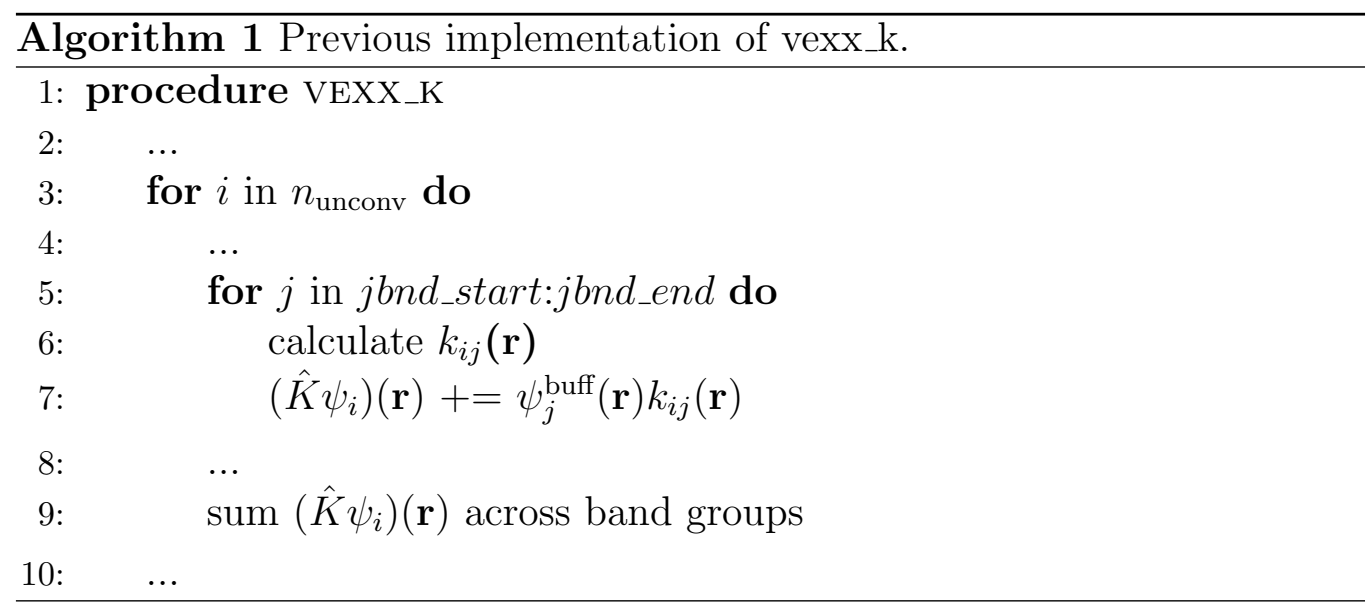

of processes, the use of some form of band parallelization is essential for the purpose of obtaining optimal performance. These results, along with all others in this paper, are obtained using the NERSC [27] Edison system, which consists of Intel Ivy Bridge nodes with dual socket 12 core E5-2695 processors. Fig. 1(a) shows the walltime of an SCF calculation on a cubic simulation cell of 64 water molecules, using the semi-local PBE functional [26] and 64 nodes. The walltimes obtained for this system are found to depend strongly on the number of task groups used, with the calculation using 4 task groups requiring approximately half the walltime of the calculation using 1 task group. Similarly, Fig. 1(b) shows the dependence of the walltime of a 64-node SCF calculation using the hybrid PBE0 functional upon the number of band groups used. When using the hybrid functional, the importance of band parallelization is especially large, with up to a $28 \mathrm{x}$ speedup being obtained when band groups are employed. From these results, it is clear that the optimal performance of QE is largely dependent upon the efficiency of the band parallelization, particularly when calculating exact exchange.

Fig. 2 shows a breakdown of the different contributions to the walltime of a 64-node SCF calculation on a cubic simulation cell of 64 water molecules, using 64 band groups. Counter-intuitively, in QE 5.2.0 the walltime associated with the local regions of the code dominates the total cost of the calculation, such that the exact exchange regions of the code only represent approximately $24 \%$ of the total walltime. This is inconsistent with the expectation described in Sec. 1.1 that the calculation of exact exchange should dominate the cost of the calculation, and is a consequence of the fact that 

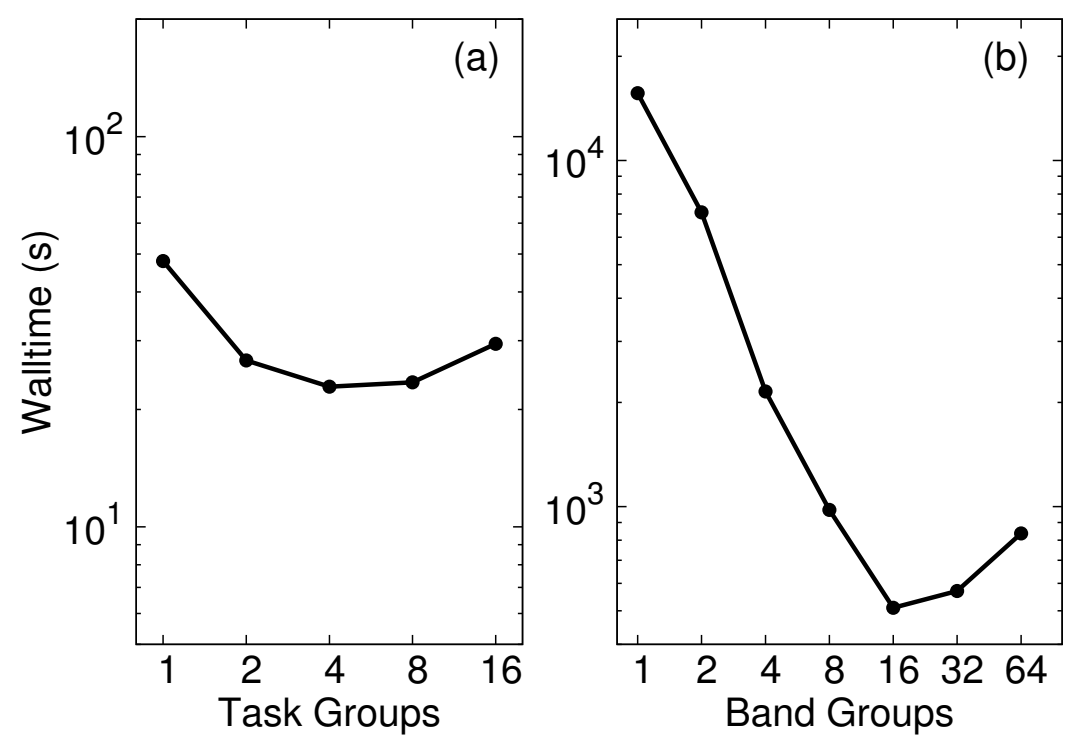

Figure 1: Walltimes for an SCF calculation on a cubic simulation cell of 64 water molecules using QE version 5.2.0, on a log-log scale. Part (a) provides results using the semi-local PBE functional for different numbers of task groups, while part (b) provides results using the hybrid PBE0 functional for different numbers of band groups. All calculations were performed using 64 Ivy Bridge nodes, with 24 MPI tasks per node. We note that in the case of our improved code described in Sec. 3, the parallelization efficiency of band groups is enhanced, leading to larger performance gains with respect to increasing number of band groups.

when using band groups, all of the work outside of the exact exchange regions of the code is duplicated across band groups. More specifically, everything outside of the inner loop of Algorithm 1 is performed serially with respect to band groups, which causes the walltime of the local regions of the code to increase linearly with respect to the number of band groups. In Sec. 3.2, we describe an algorithm for parallelizing the calculation of the local regions of the code between band groups, which reduces the walltime associated with the local regions of the code and leads to the more intuitive results shown by the blue columns in Fig. 2.

Fig. 3 shows the fraction of walltime associated with the local regions of the code for several SCF calculations performed for systems of 16, 32 and 64 water molecules, and using the PBE0 functional and various numbers of nodes. In each calculation, the number of band groups is equal to the number of nodes. The fraction of time spent in the local part of the calculation 


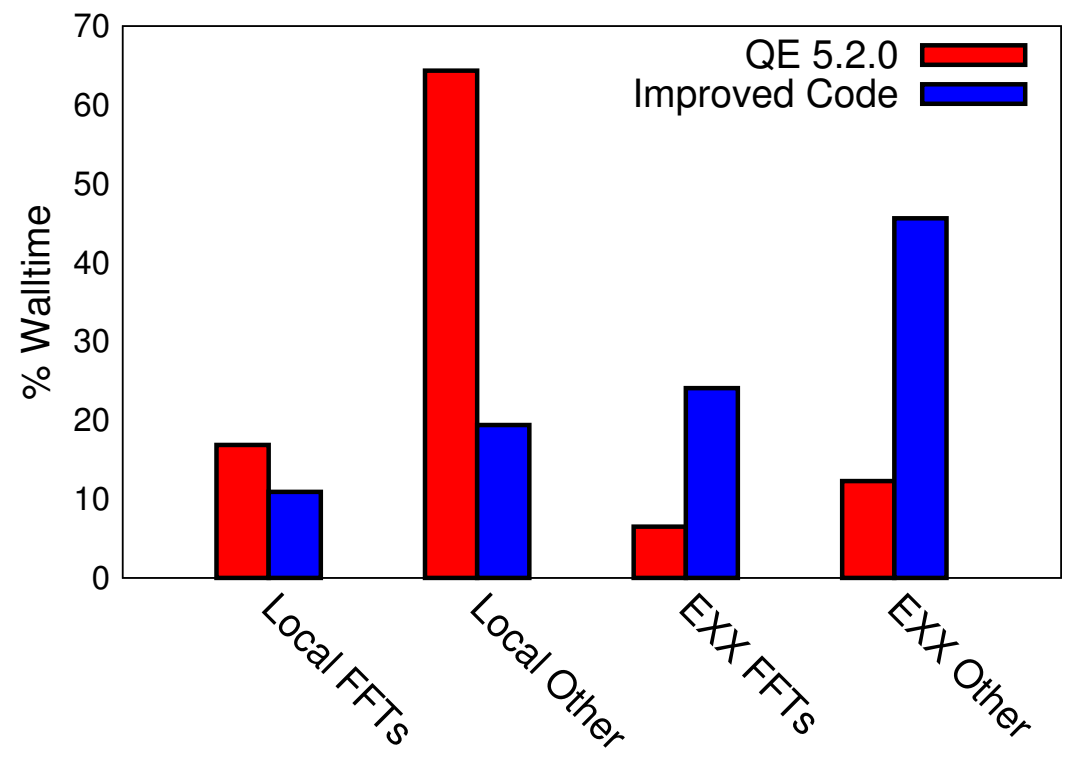

Figure 2: Relative contributions to the walltime of an SCF calculation on a system of 64 water molecules, using 64 Ivy Bridge nodes and 64 band groups. From left to right, timings are reported for the FFTs performed in local regions of the code, all other work in the local regions of the code, the FFTs performed in the exact exchange regions of the code, and all other work performed in the exact exchange regions of the code. Using QE 5.2.0, a majority of the calculation time is spent in the local regions of the code. Using the improvements described in Sec. 3, a much smaller amount of time is spent on the local regions of the code, resulting in a larger proportion of time being spent in the exact exchange regions of the code.

increases with the number of nodes; moreover, it is observed that regardless of the size of the system, the relative cost of the local part of the calculation is largely determined by the ratio of the number of nodes to the number of molecules. Because practical calculations on large systems tend to require using correspondingly large numbers of nodes and band groups, the fraction of time spent in the local regions of the code does not necessarily decrease as the size of a system is increased. In Sec. 3.2 we propose a method for parallelizing the exact exchange regions of the code independently from the parallelization of the local regions of the code, the local regions of the code can be parallelized between band groups. 


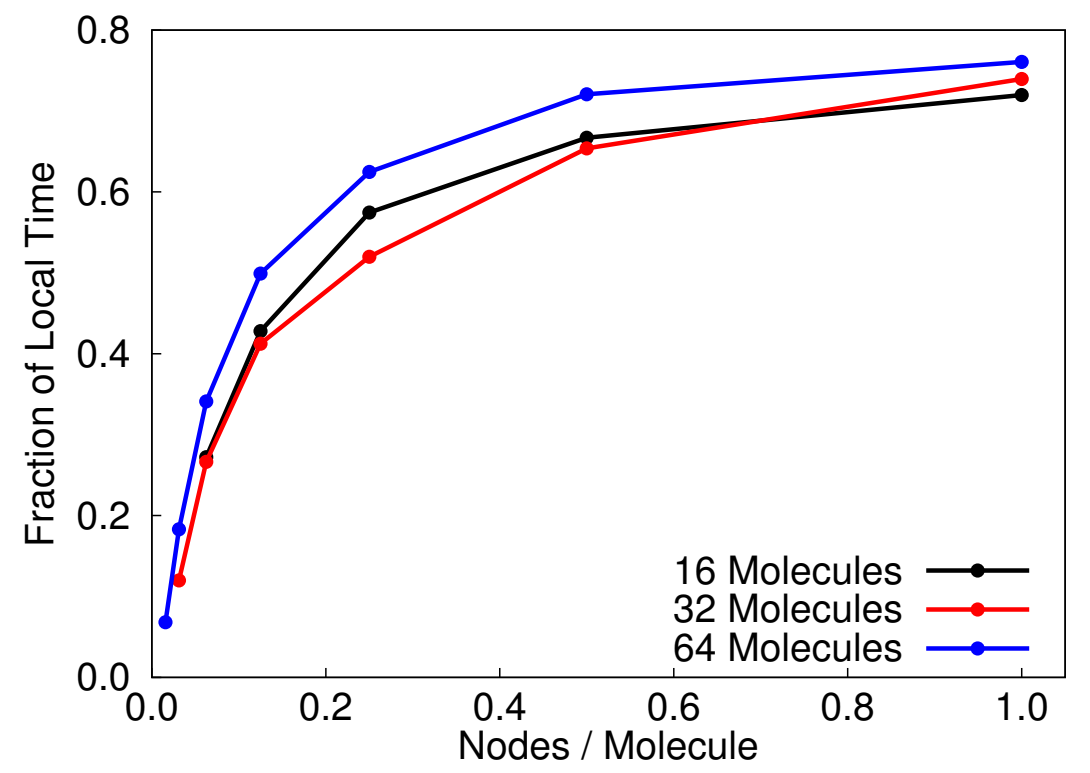

Figure 3: Fraction of walltime spent in local regions of the code for an SCF calculation performed on systems of 16,32, and 64 water molecules, and using QE 5.2.0. Each calculation is performed using 1 band group per node. For any given ratio of the number of nodes to the number of molecules, the fraction of time spent in local regions of the code is largely independent of the total simulation size. Furthermore, the fraction of time spent in local regions of the code increases as more nodes are used.

\section{Code Improvements}

As illustrated by Fig. 4, calculation of the exact exchange contribution to $H \psi$ involves two loops over bands; however, in previous versions of QE only the inner loop is parallelized with respect to band groups. In Sec. 3.1, we describe a modified approach to band group parallelization in which instead of parallelizing over bands in the inner loop, the parallelization is performed over individual band pairs. In addition to eliminating duplication of work in "Loop i" of Fig. 4, this approach improves load balancing and significantly increases the scalability of the code. In Sec. 3.2, we describe a method for enabling parallelization of the work in the "Diag. Loop" of Fig. 4 across band groups. This method requires a transformation of the data structure representation of $\psi_{i}$ and $\hat{K} \psi_{i}$, which is illustrated by Fig. 5 and discussed in more detail in Sec. 3.3. 

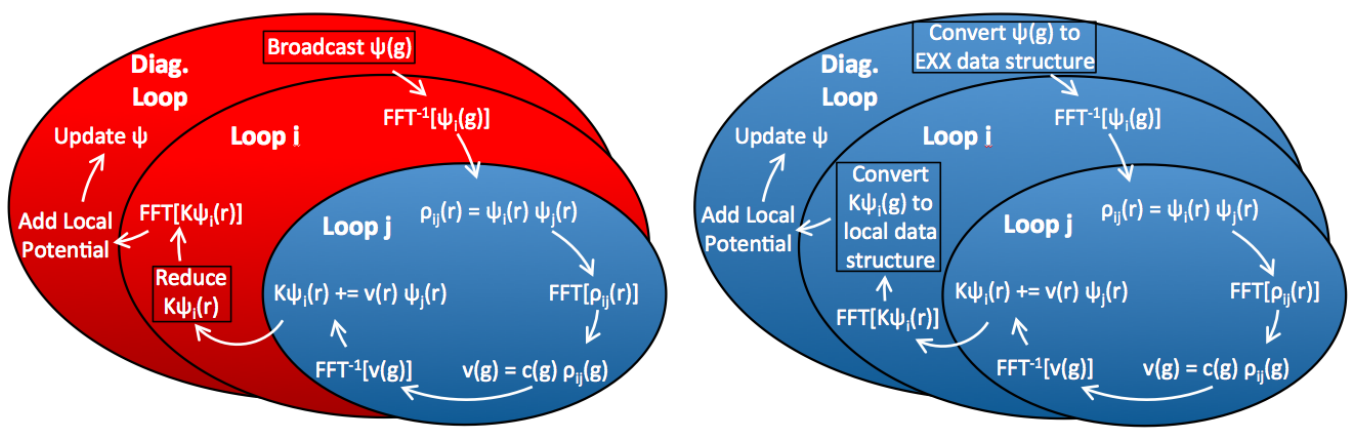

Figure 4: Overview of the code in QE 5.2.0 (left) and with the improvements described in Sec. 3 (right). Each oval represents a loop within the SCF calculation. The "Diag. Loop" corresponds to the iterative Davidson diagonalization algorithm, "Loop i" corresponds to the outer loop over bands in subroutine vexx_k, and "Loop j" corresponds to the inner loop over bands in subroutine vexx_k. Loops that are performed serially with respect to band groups are colored red, while loops that are performed in parallel with respect to band groups are colored blue. Steps involving inter-band group communication are highlighted by a black border. Major changes in the improved code include the introduction of band group parallelization in Loop i, as discussed in Sec. 3.1, and the parallelization of the local part of the code across band groups, as discussed in Sec. 3.2. Enabling parallelization of the local part of the code across band groups requires on-the-fly changes to the data structure of $\psi_{i}$ and $\hat{K} \psi_{i}$, as described in Sec. 3.3 and depicted in Fig. 5.

\subsection{Pair Parallelization}

As illustrated by the data in Fig. 6, load balancing in previous versions of QE becomes problematic for large $n_{\text {bgrp }}$. The timings are obtained from a system of 16 water molecules having a total of 64 doubly-occupied valence bands. When $n_{\text {bgrp }}$ is within approximately an order of magnitude of the number of occupied bands, the strong scaling profile of QE exhibits discontinuities caused by imperfect load balancing in Algorithm 1. Because each band group is assigned an integer number of bands in the inner loop of Algorithm 1, perfect load balancing is only possible when the number of bands is evenly divisible by the number of band groups. As $n_{\text {bgrp }}$ increases, the workload becomes increasingly imbalanced between the band groups. A maximum of $n_{\text {bnd }}$ band groups can be employed, as any additional band groups would have a load of zero bands.

An alternative approach to the parallelization over bands described in Algorithm 1 is instead to parallelize over band pairs, as described in Algorithm 2. Because the number of band pairs is equal to $n_{\text {bnd }} n_{\text {unconv }}$, it is possible to distribute band pairs more evenly between band groups. In this 
Local Data Structure of $\boldsymbol{\Psi}_{i}(\mathrm{~g})$

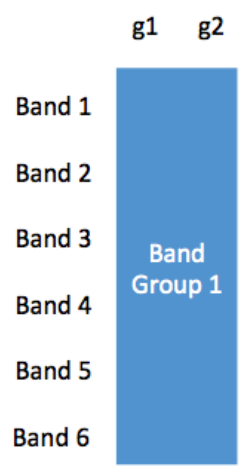

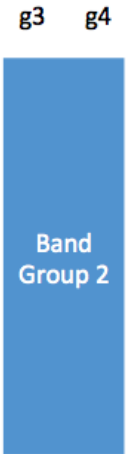

g5 g6

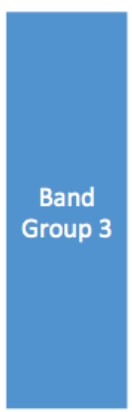

EXX Data Structure of $\boldsymbol{\Psi}_{i}(\mathrm{~g})$

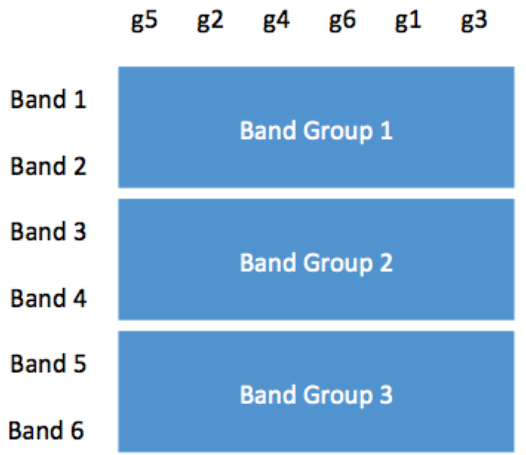

Figure 5: Data structure representation of $\psi_{i}$. As shown in Fig. 4, independent parallelization of the local and exact exchange regions of the code requires an on-the-fly transformation of the data structure of $\psi_{i}$ and $\hat{K} \psi_{i}$ from a representation that is suitable for the local regions of the code and another representation that is suitable for the exact exchange regions of the code. This is because the parallelization in the local regions of the code is implemented according to the convention that $\left\{\psi_{j}\right\}$ is distributed between nodes in the manner indicated on the left, with each band being distributed across all nodes. Conversely, band-pair parallelization of the exact exchange calculation requires that each band group stores the entirety of $\left\{\psi_{j}\right\}$, but only for a subset of bands. Transformation between these two data structures is conceptually similar to a transpose; however, because the ordering of the g-vectors is not the same in each case, the actual process is significantly more complex. Details of the data structure transformation algorithm are provided in Sec. 3.3 .

approach, the outer loop is parallelized with respect to band groups, with the variables ibnd_start and ibnd_end performing roles analogous to jbnd_start and jbnd_end in Algorithm 1. For the inner loop of vexx_k, the values of $j b n d \_s t a r t$ and $j b n d \_e n d$ depend upon $i$. Because $n_{\text {unconv }}$ is not constant between iterations of the Davidson diagonalization algorithm, the total number of pairs that are calculated by Algorithm 2 is not the same each time the algorithm is executed. As a result, the values of $i b n d \_s t a r t, i b n d \_e n d, j b n d \_s t a r t$, jbnd_end are updated at the start of the algorithm. In order to minimize the amount of duplicated work in the outer loop, pairs are assigned to band groups in such a way as to minimize the range of ibnd_start : ibnd_end for each band group; thus, in addition to improving load balancing, Algorithm 2 also reduces the amount of code that is performed serially with respect to band groups. 


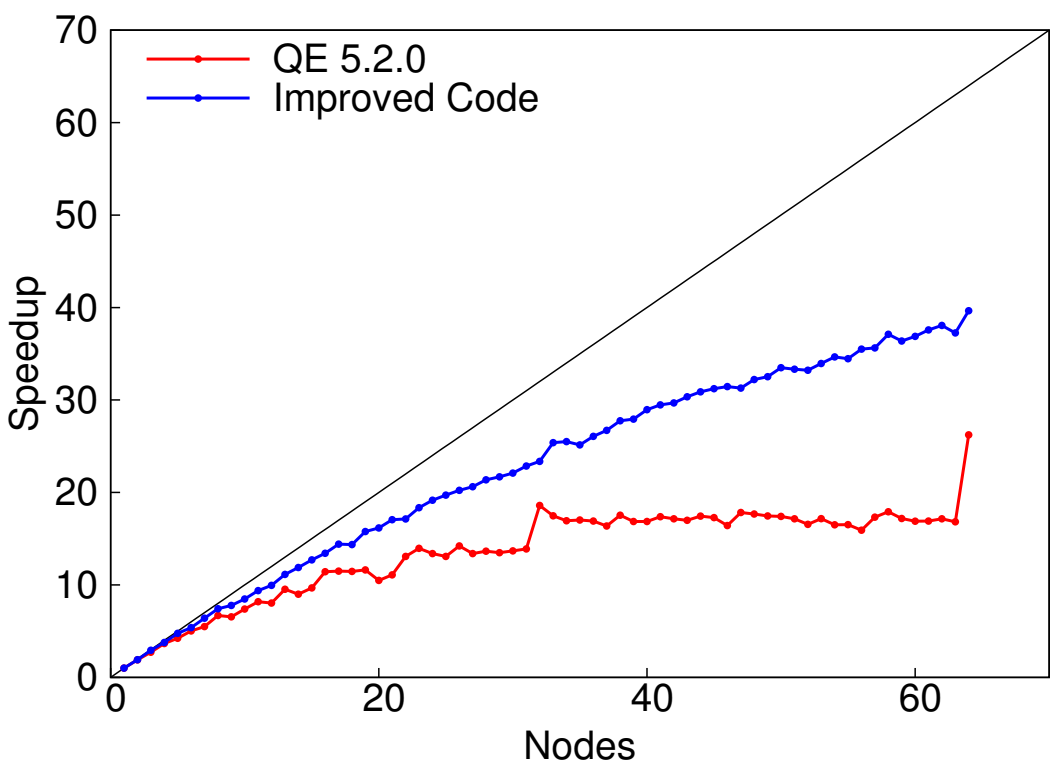

Figure 6: Strong scaling of an SCF calculation on a system of 16 water molecules using the PBE0 functional. Timings are collected only for the calls to vexx, and without inclusion of any inter-band group communication operations within vexx; these communication costs are discussed in Sec. 3.3. The red curve corresponds to QE 5.2.0, while the blue curve corresponds to the band-pair parallelization approach. Ideal scaling is shown by the black lines. The large jumps in the QE 5.2.0 timings at 32 nodes and 64 nodes are a result of imperfect load balancing.

\subsection{Independent Parallelization of the Exact and Local Regions of the Code}

As described in Sec. 2.2, one of the primary inefficiencies in the use of band group parallelization is the duplication of the local part of the calculation across band groups. In this section, we describe a strategy for enabling independent parallelization of the local and exact exchange regions of the code, such that the use of band groups does not affect the parallelization or performance of the local regions of the code.

The key change involved in our independent parallelization scheme is that in the local regions of the code, the calculation is performed as though the DFT functional was non-hybrid and $n_{\text {bgrp }}=1$, regardless of the actual value of $n_{\text {bgrp }}$ input by the user. Consequently, the local part of the calculation is always parallelized across all $n_{\text {nodes }}$ used by the calculation, with the same performance that would be obtained if $n_{\text {bgrp }}=1$. Furthermore, this change enables the use of task groups for the computation of FFTs in the local 


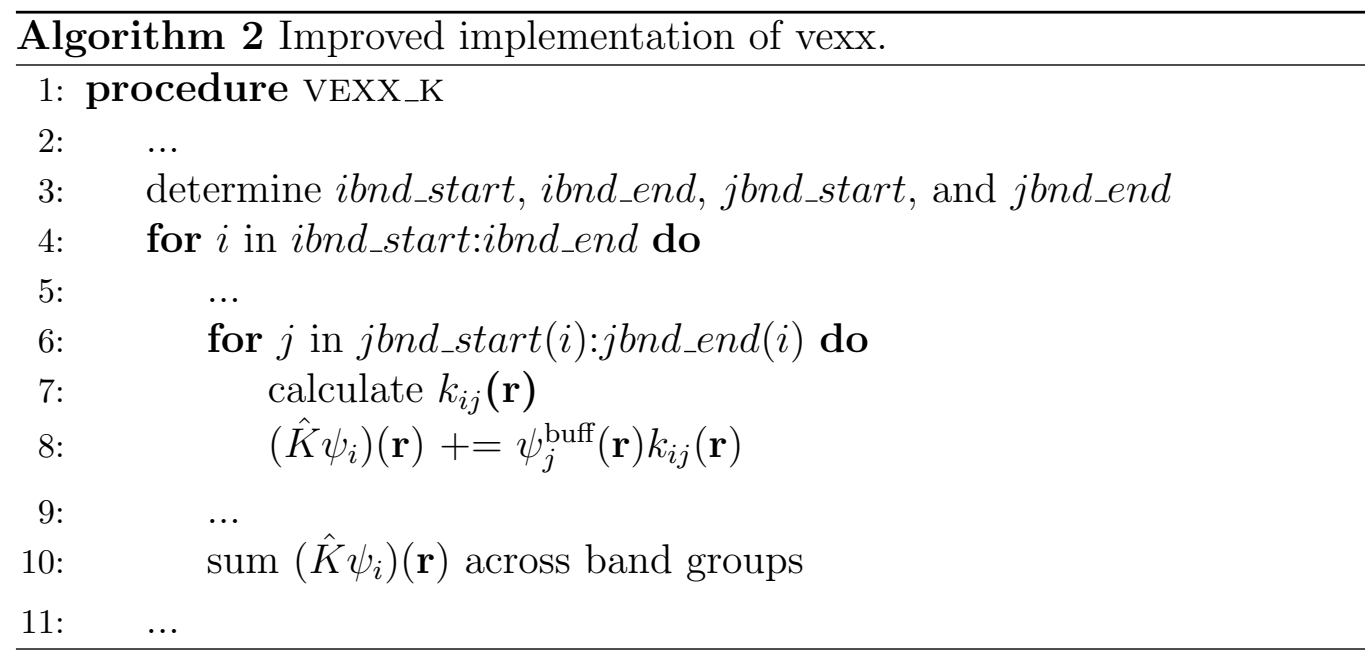

regions of the code, which was shown in Sec. 2.2 to be essential for obtaining good performance when parallelizing across large numbers of nodes. In the exact exchange regions of the code, the calculation is performed using the user-defined value of $n_{\text {bgrp }}$, similarly to how this part is normally performed in QE 5.2.0, except with the additional improvements described throughout this paper.

The primary challenge involved in implementing this independent parallelization method is that it requires that the data structure representation of several quantities, including $\psi_{i}(g)$ and $H \psi_{i}(g)$, be different in the local and exact exchange regions of the code. In the local regions of the code, $\psi_{i}(g)$ and $H \psi_{i}(g)$ are distributed across all processes with respect to g-vectors, with every process having a subset of the g-vectors for all bands $i$. In the exact exchange regions of the code, $\psi_{i}(g)$ and $H \psi_{i}(g)$ are distributed across all processes within a single band group, with the processes in a single band group storing values for only a subset of the bands $i$.

Fig. 5 illustrates the difference between the local and exact exchange data structures. Transformation between the two data structures approximately corresponds to a transpose operation; however, the process is greatly complicated by the fact that each data structure utilizes a different ordering of the g-vectors. As a result, transforming between the local and exact data structures is algorithmically non-trivial, and the performance and scalability of our independent parallelization method is largely dependent on the efficiency of this process. The implementation details of this transformation are 
described in the next section.

\subsection{Data Structure Transformation}

For each iteration of the iterative Davidson diagonalization algorithm, the exact exchange potential, $\hat{K} \psi$, is calculated through a call to the subroutine vexx_k. Within the context of the independent parallelization approach described in Sec. 3.2, every call to vexx_k requires two transformations of the data structure. First, at the start of vexx_k, $\psi$ must be transformed from the local data structure to the exact exchange data structure. Second, at the end of vexx_k, $\hat{K} \psi$ must be transformed from the exact exchange data structure to the local data structure.

Algorithm 3 describes the transformation from the local data structure to the exact exchange data structure. There are three main steps in this process. First, an inter-band group communication step is performed in which each band group gathers all of the data that it requires from all of the other band groups. Second, an intra-band group communication step is performed in which each process within a band group gathers all of the data that it requires from all of the other processes within the band group. Third, each process places the gathered data into the correct locations in the transformed $\operatorname{array} \psi_{i}^{\operatorname{exx}}$

Importantly, the entire transformation in Algorithm 3 requires the com-

munication of at most $4 n_{\mathrm{g}}$ ceil $\left[\frac{n_{\text {bnd }}}{n_{\text {bgrp }}}\right]$ floats per band group, where $n_{\mathrm{g}}$ is the number of g-vectors. This is an improvement over previous versions of QE, for which the calculation of the exact exchange potential involves communication steps that exhibit poor strong scaling. In previous versions that duplicate the work of calculating $\psi$, it is necessary to synchronize the values of $\psi$ calculated by each band group by broadcasting $\psi$ from a single band group. The broadcast requires that each band group receives a total of $2 n_{\mathrm{g}} n_{\text {bnd }}$ floats.

The transformation of $\hat{K} \psi$ at the end of vexx_k is performed similarly. First, an inter-band group communication step is performed in which the calculated contributions to each $\hat{K} \psi_{i}$ are summed across band groups. Second, a communication step is performed in which each process within a band group gathers all of the data that it requires from all of the other processes. Third, each process places the gathered data into the correct locations in the transformed array $\hat{K} \psi_{i}^{\text {loc }}$

In previous versions of $\mathrm{QE}$, the parallelization of the inner loop of vexx_k 


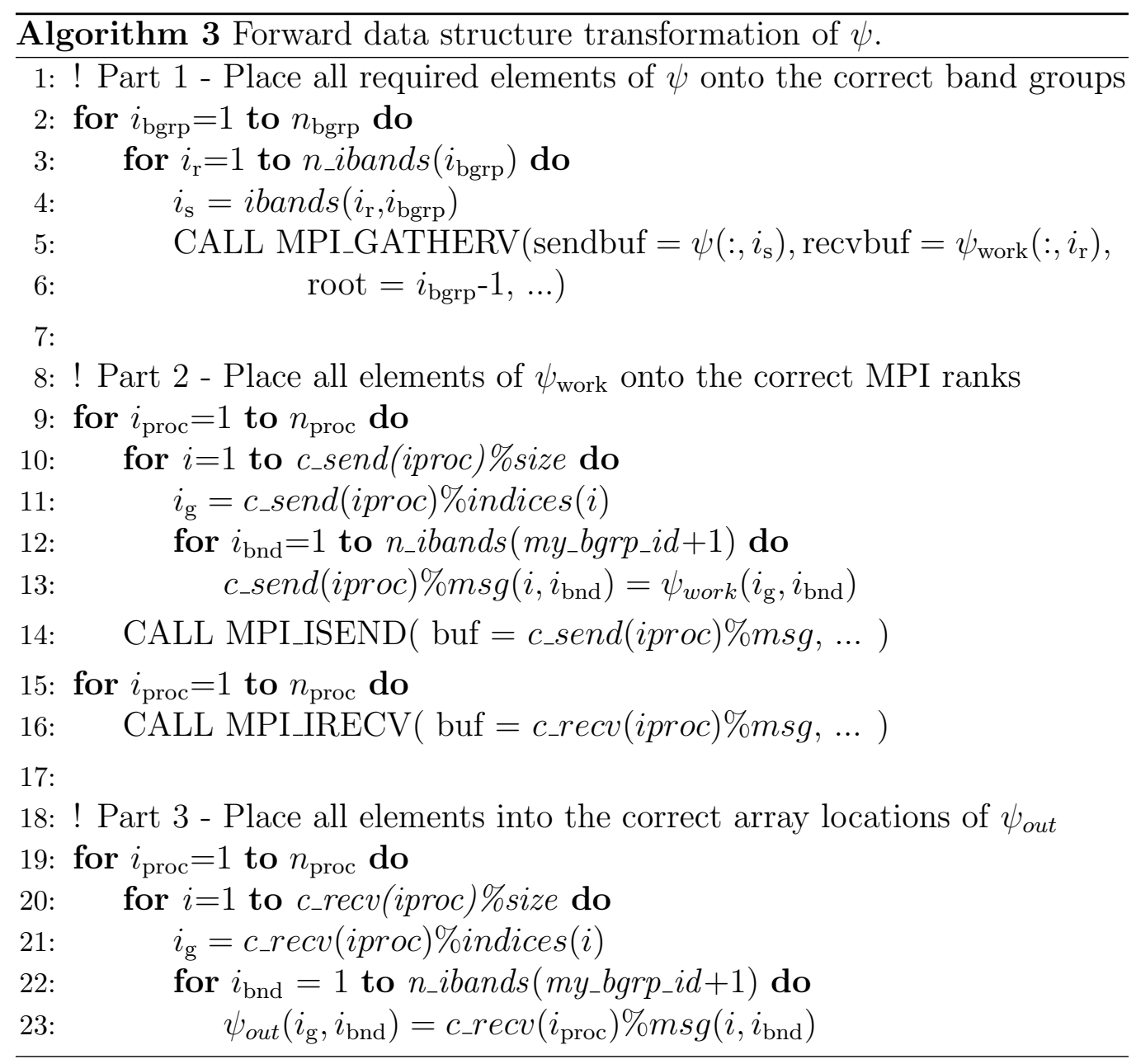


means that each band group calculates a subset of the terms required to determine each $\hat{K} \psi_{i}$; consequently, it is necessary to sum the contributions to each $\hat{K} \psi_{i}$ from all band groups. In the pair-parallelized approach described in Sec. 3.1, only a subset of the band groups will contribute to the calculation of any given $\hat{K} \psi_{i}$, greatly reducing the amount of communication required to calculate $\hat{K} \psi$. In the case in which $n_{\text {bnd }}$ is divisible by $n_{\text {bgrp }}$, all terms required to compute any given $\hat{K} \psi_{i}$ are determined by a single band group, and no inter-band group summation is necessary. Otherwise, if $n_{\text {bgrp }}$ is less than $n_{\text {bnd }}$, at most two band groups contribute to the calculation of each $\hat{K} \psi_{i}$, and a maximum of $2 n_{\mathrm{g}}$ floats must be summed across each band group.

In addition to the transformation of $\psi$ and $\hat{K} \psi$ in vexx_k, it is also necessary to transform the array evc at the beginning of the subroutine exxinit, which contains the eigenvectors that are used to construct $\psi_{j}^{\text {buff }}(\mathbf{r})$ in Algorithm 2. This transformation is performed in the same way as the transformation of $\psi$. Because exxinit is only called once per Davidson diagonalization, the transformation of evc occurs comparatively infrequently, and contributes little to the total communication costs.

Fig. 7 shows the total cost of communication required in subroutine vexx_k for an SCF calculation on a cubic simulation cell of 64 water molecules. When using QE 5.2.0, vexx_k requires the use of both an inter-node broadcast and an inter-node summation; consequently, the cost of communication increases with respect to the number of nodes used. When using the improved parallelization scheme presented in this paper, the total amount of data that must be communicated within vexx_k is much smaller, and tends to decrease with respect to the number of band groups; this results in significantly improved communication costs. Further optimizations would be possible for large calculations and for machines with good support for overlapped computation and parallel communication, because computation of vexx_k could commence on subsets of the data, before the entire data set was transferred.

\section{Conclusions and Outlook}

In this paper, we have presented an improved method for the parallelization of hybrid DFT calculations within QE. As shown in Fig. 8(a), these changes lead to large improvements in the strong scaling of the code. In particular, the cost of a 96-node calculation on a system of 64 water molecules is

found to be a factor nine times cheaper and faster than in previous versions of 


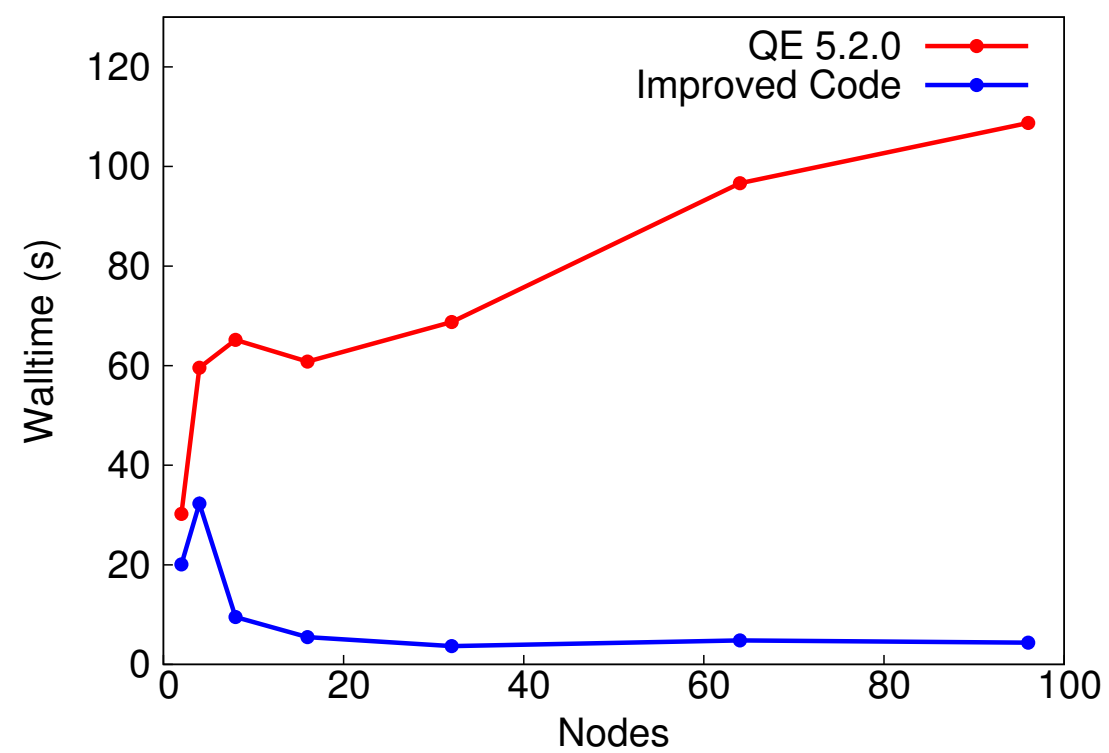

Figure 7: Total walltimes for the communication operations performed within the subroutine vexx_k. All calculations are performed using 1 band group per node. The red curve shows the cost of communication using QE 5.2.0, while the blue curve shows the cost of communication using the modifications described in this paper.

the code. This approximately order-of-magnitude enhancement is made possible through the combination of a band-pair approach to load distribution and an efficient algorithm for independently parallelizing the exact exchange and local regions of the code, which enables the use of band groups and task groups within a single execution of the code. We are currently working to incorporate the improved algorithm described here in the 6.x releases of QE.

Interestingly, Fig. 8(b) shows that the strong scaling of subroutine vexx_k is superior to the strong scaling of the overall SCF code. This suggests that when using our algorithm, the parallelization efficiency of a hybrid DFT calculation is primarily limited by the performance of the local regions of the code. Further improvements to the strong scaling are thus likely to require refactoring of the local regions of the code, likely with special attention to the implementation of task groups. 


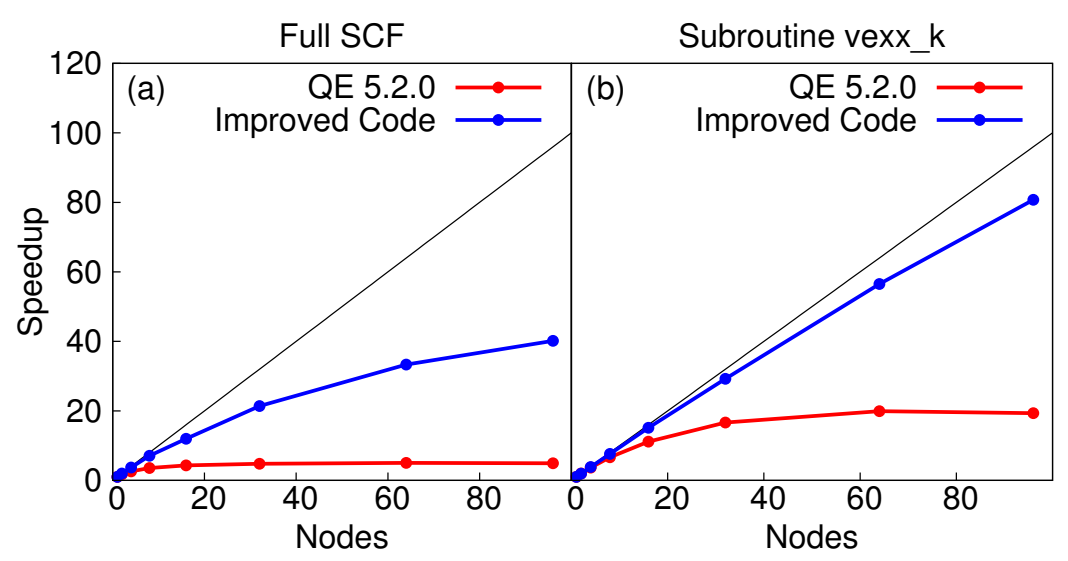

Figure 8: Strong scaling of the code. (a) Full SCF calculation. (b) Subroutine vexx_k only. All calculations are performed on a system of 64 water molecules. The red curve corresponds to QE 5.2.0, while the blue curve corresponds to the improved code described in this paper. Ideal scaling is shown by the black lines.

\section{Acknowledgements}

This research used resources of the National Energy Research Scientific Computing Center, a DOE Office of Science User Facility supported by the Office of Science of the U.S. Department of Energy under Contract No. DEAC02-05CH11231.

Work by PK was conducted at the Center for Nanophase Materials Sciences, which is a DOE Office of Science User Facility.

Work by DP was conducted at The Molecular Foundry, which is supported by the Office of Science, Office of Basic Energy Sciences, of the United States Department of Energy under Contract No. DE-AC02-05CH11231.

We thank Christopher Dahnken, Hans Pabst, and Thanh Phung for useful discussions.

\section{References}

[1] P. Hohenberg, W. Kohn, Phys. Rev. 136 (1964) B864.

[2] W. Kohn, L.J. Sham, Phys. Rev. 140 (1965) A1133.

[3] J. Landers, G.Y. Gor, A.V. Neimark, Colloids and Surfaces A: Physicochemical and Engineering Aspects 437 (2013) 3. 
[4] A. Zangwill, Physics Today 68 (2015) 34.

[5] J.K. Norskov, F. Abild-Pedersen, F. Studt, T. Bligaard, PNAS 108 (2011) 937.

[6] J. Klimes, A. Michaelides, J. Chem. Phys. 137 (2012) 120901.

[7] A.J. Cohen, P. Mori-Sánchez, W. Yang, Science 321 (2008) 792.

[8] P. Mori-Sánchez, A.J. Cohen, W. Yang, Phys. Rev. Lett. 100 (2008) 146401.

[9] J. Heyd, G.E. Scuseria, M. Ernzerhof, J. Chem. Phys. 118 (2003), 82078215 .

[10] J. Heyd, G.E. Scuseria, M. Ernzerhof, J. Chem. Phys. 124 (2006), 219906.

[11] A.D. Becke, J. Chem. Phys. 98 (1993) 5648.

[12] J.P. Perdew, M. Ernzerhof, K. Burke, J. Chem. Phys. 105 (1996) 99829985.

[13] C.J. Cramer, D.G. Truhlar, Phys. Chem. Chem. Phys. 11 (2009) 10757.

[14] R. Baer, E. Livshits, U. Salzner, Annu. Rev. Phys. Chem. 61 (2010) 85.

[15] J.P. Perdew, A. Ruzsinszky, L.A. Constantin, J. Sun, G.I. Csonka, J. Chem. Theory Comput. 5 (2009) 902.

[16] K. Burke, J. Chem. Phys. 136 (2012) 150901.

[17] W.J. Cohen, P. Mori-Sánchez, W. Yang, Chem. Rev. 112 (2011) 289.

[18] A.D. Becke, J. Chem. Phys. 136 (2014) 18A301.

[19] R.A. DiStasio, Jr, B. Santra, Z. Li, X. Wu, R. Car, J. Chem. Phys. 141 (2014) 084502.

[20] Q. Wan, L. Spanu, F. Gygi, G. Galli, J. Phys. Chem. Lett. 5 (2014) 2562.

[21] L. Lin, JCTC. 12 (2016) 2242. 
[22] E.J. Bylaska, K. Glass, D. Baxter, S.B. Baden, J.H. Weare, Journal of Physics: Conference Series 180 (2009) 012028.

[23] C. Zhang, D. Donadio, F. Gygi, G. Galli, J. Chem. Theory Comput. 7 (2011) 1443.

[24] I. Duchemin, F. Gygi, Comp. Phys. Comm. 181 (2010) 855.

[25] P. Giannozzi, S. Baroni, N. Bonini, M.Calandra, R.Car, C.Cavazzoni, D.Ceresoli, G.L. Chiarotti, M. Cococcioni, I. Dabo, A. Dal Corso, S. de Gironcoli, S. Fabris, G. Fratesi, R. Gebauer, U. Gerstmann, C. Gougoussis, A. Kokalj, M. Lazzeri, L. Martin-Samos, N. Marzari, F. Mauri, R. Mazzarello, S. Paolini, A. Pasquarello, L. Paulatto, C. Sbraccia, S. Scandolo, G. Sclauzero, A.P. Seitsonen, A. Smogunov, P. Umari, R.M. Wentzcovitch, J. Phys.: Condens. Matter. 21 (2009) 395502 (19pp).

[26] J.P. Perdew, K. Burke, M. Ernzerhof, Phys. Rev. Lett. 77 (1996) 3865.

[27] NERSC: http://www.nersc.gov

[28] http://www.fftw.org 